Melzer, D., Hale, A. S., Malik, S. J., et al (1991) Community care for patients with schizophrenia one year after hospital discharge. British Medical Journal. 303, 1023-1026.

University of Sheffield Medical School

JIM BODDINGTON

Beech Hill Road

Sheffield S10 2RX

\section{Developing training themes from HRH's delivery}

SIR: The Collegiate Trainees Committee welcome HRH The Prince of Wales's interest and depth of understanding in a thoughtful and provoking address to the College on its 150th anniversary (Journal, December 1991, 159, 763-768). The Committee wishes to develop some of the Prince's comments, particularly in relation to training.

We welcome HRH's emphasis on the holistic approach to healing and the need to emphasise the physical, mental and spiritual aspects of healing in the training of doctors in general and psychiatrists in particular. Religious and spiritual factors influence the experience and presentation of illness and a multi-ethnic perspective is essential in the management of psychiatric disorders in a multicultural society. This should be reflected in psychiatric training and, although many psychiatric units have close links with representatives of different religions and cultures, few psychiatric training schemes involve a formal input.

In the current climate of health care in Britain, we are concerned about diminishing opportunities for psychiatric trainees to learn from the specialist skills of other allied professionals, mainly as a result of reduced numbers of psychologists, social workers, and occupational therapists in psychiatric services. We welcome the Prince's comments on closer liaison between health and social services and see such joint work and training as helpful in developing a more holistic approach.

HRH highlighted the division between biologically orientated psychiatrists who were over-reliant on physical and chemical modes of treatment, and psychotherapists who solely use psychological treatments. We think it is important that such an impression must be seen against a backdrop of limited resources - especially for the development of psychotherapeutic treatments within the National Health Service. Good training enables an eclectic approach, but poor training and time restraints may lead to more 'medication focused' consultations, hence further distancing the psychiatrist and his or her patient from an holistic approach.

HRH identified the lack of public exposure to mental illness in the media. We consider that the potential for misportrayal of mental health issues and psychiatric practice exceeds that of many other branches of medicine. We feel that psychiatrists as a profession should be prepared to be more active in media liaison and we are concerned that presentation skills, from conducting a case conference through to lecturing and media skills, have been largely ignored in the training of psychiatrists. We understand that the Public Education Committee of the College is attempting to address some of these problems by teaching psychiatrists how to talk to the media.

HRH brought attention to several areas of changing service provision which have important implications for psychiatric training. With increasing numbers of chronically ill patients being discharged from long-term beds and fewer beds being available for acute care, an increase in emergency presentations to psychiatric hospitals, accident and emergency departments and police services is to be expected (Department of Health, 1989). Emphasis thus needs to be given in training on how to manage psychiatric emergency consultations and, in planning such services, the supervision and safety of trainees who man them is a priority. Psychiatric training must address the changing needs of the community and its health service. Community psychiatry, liaison with general practitioners, closer work with social work departments, and the voluntary sector all require greater attention. Closer training links between general practitioners and psychiatrists will be of help, and such links should be reciprocal. We would also encourage trainees to visit and learn about models of service practised in other areas of the country and beyond.

We hope that psychiatrists will not ignore the challenges put forward by HRH's address.

Department of Health (1989) Caring for People. London: HMSO.

R. KEHOE
A. MOORE
J. PEARCE
M. WARD
F. JAMIESON
J. QUINN
Working Group, Collegiate Trainees Committee
The Royal College of Psychiatrists
17 Belgrave Square
London SWIX $8 P G$

SIR: I would like to add some comments to the wise remarks of the HRH The Prince of Wales (Journal, December 1991, 159, 763-768) regarding the current and future roles of psychiatrists. Most of us are aware of the biopsychosocial theoretical framework 
for medical science (Engel, 1980), and our Patron has drawn attention to potentially additional dimensions of spiritual, ecological, and perhaps even political influences on the human psyche and its manifestations. The variety and complexity of these influences is a source of both wonder and frustration; it is the nature of science to attempt to reduce phenomena to their fundamentals, and the recent surge of interest in neurobiology holds much promise for the alleviation of mental suffering. However, no therapeutically significant achievements have as yet been realised from this approach and it would be foolish to replace an historical overzealous application of psychoanalytical theories with a similarly extreme, premature bias towards the biological in psychiatry.

The Prince warns of the implications of our medical model and materialistic viewpoint towards mental illness and psychiatric training. We should remember that medical training distances us from emotional involvement with patients (the lay sense of the word 'clinical' is of a cold, detached attitude) which is reinforced by an increasing reliance on drug treatments. The dictates of time, pressure of work and shortage of resources discriminate against the time-consuming psychotherapies which can affect a more meaningful and longer-term benefit for the patient. The need for trainees to produce research publications impels them to undertake brief, often ill-conceived and ultimately inconsequential, shortsighted projects with a view to personal gain through promotion. Biological studies are appealing to psychiatrists at all stages of their careers as they tend to attract greater funding, demand fewer numbers of subjects, and are quicker to conduct than those of a psychosocial orientation - important factors when one considers the 'publish or perish' phenomenon where quantity is often valued over quality. Such strong motivators, the medical hierarchial career structure, and the need to emulate our mentors all encourage the expression of a simplistic biological reductionism that could skew our training and clinical practice, and may even hinder further development in the understanding of psychiatric conditions and their treatment. Continued research is essential in advancing the frontiers of our knowledge, but we must bear in mind that up to three-quarters of the scientific literature may be worthless (Hamilton, 1990) and conventional limits of statistical significance are arbitrarily determined.

His Royal Highness sees the future of psychiatry in the community and in prevention. We must retain a flexibility and balance in our approach, or we risk losing ground to other professionals and nonstatutory agencies; even our raison d'etre (Lancet,
1985). Unless we are to ignore the vast majority of emotional distress and psychological disorder we shall have to be adept in counselling and psychotherapeutic skills, or at least the supervision of them. It is of concern that there are no requirements for psychotherapeutic experience during our training. Our conceptions of psychiatric illness are city and institution based, where people can easily be depersonalised and dehumanised. We should remember that we are employed to provide a service to our patients. To return them to their GP with the comment "no formal psychiatric illness discharge" is a dismissive, unhelpful practice to be condemned. The vagaries of the human condition are such that it is unlikely we shall ever be able to sharply demarcate distress from illness, however defined.

To be provocative: I wonder how much biological psychiatry is a determined attempt to enhance credibility with our medical colleagues or to remove us from scientific and political controversy surrounding social issues, how different things might be if even some of the vast expenditure on drugs and research in the past thirty years had been invested in combating social adversity, how ecologically damaging the processes and effects of drug manufacture are, and whether psychiatrists should be more overtly political in challenging the effects of social deprivation on mental health. To my mind our male-dominated, scientific, mechanistic view of mental illness needs to include a more feminine, subjective and sensitive aspect.

ENGEL, G. L. (1980) The clinical application of the biophsychosocial model. American Journal of Psychiatry, 137, 535-544.

Hamilton, D. P. (1990) Publishing by-and for?-the numbers. Science, 250, 1331-1332.

LANCET (1985) Psychiatry - discipline that has lost its way. Lancet, $i$ $731-732$.

Royal Edinburgh Hospital Edinburgh EHIO SHF

STEPHEN M. LAWRIE

\section{Use of fluoxetine in heroin addiction}

SIR: A priority in the treatment of heroin addiction is the prevention of relapse (Guelfi \& Trampetti, 1989). The use of opiate antagonist drugs has been advised to control relapse, following Wikler's 'deconditioning' theory (Wikler, 1974). Unfortunately, the results obtained by opiate antagonists are unsatisfactory and many patients discontinue treatment and continue with heroin. Only patients who have strong personal motivation show positive results with this method (Greenstein et al, 1976). 